\title{
Regional Metabolic Assessment of Human Brain during Development by Proton Magnetic Resonance Spectroscopy In Vivo and by High-Performance Liquid Chromatography/Gas Chromatography in Autopsy Tissue
}

PETRA S. HÜPPI, CHRISTOPH FUSCH, CHRIS BOESCH, ROLAND BURRI, EMILIO BOSSI, MAURIZIO AMATO, AND NORBERT HERSCHKOWITZ

Department of Pediatrics and Division of Magnetic Resonance Spectroscopy, University of Berne, Berne, Switzerland

\section{ABSTRACT}

To study the course of regional metabolite concentrations during early brain development, we measured in vivo metabolites [Nacetyl-aspartate (NAA), choline-containing compounds, and myoinositol (M-Ino)] in the precentral area of the cerebrum by short echo-time single volume proton magnetic resonance spectroscopy and compared in vivo established spectroscopic data with classic chromatographic data (HPLC) on age-corresponding autopsy tissue in different regions of the brain. In autopsy tissue, regional (frontal lobe, precentral area, basal ganglia, thalamus) and age-dependent differences of the concentration of creatine, NAA, and M-Ino were determined. In vivo measurement of NAA by proton magnetic resonance spectroscopy shows a significant increase of NAA by increasing postconceptional age. M-Ino shows a weak correlation and a nonsignificant decrease with increasing postconceptional age. Choline shows no age-dependent changes. Creatine concentrations measured by HPLC in different regions of the developing brain at autopsy showed an age-dependent increase that was identical for the left and right side and similar for the precentral area and frontal lobe and more pronounced for the basal ganglia and thalamus. Comparison of the results obtained by the two methods shows agreement for the age-dependent changes and the absolute concentration of M-Ino. NAA determined in autopsy tissue by HPLC is significantly lower than that measured in vivo by proton magnetic resonance spectroscopy. A comparison of the concentrations measured by HPLC in frontal lobe, basal ganglia, and thalamus with the results obtained from the precentral area showed significant regional differences in all measured metabolites. These results define important agedependent changes detected with both methods and further indicate limitations of both methods that have to be considered when presenting absolute concentration values. (Pediatr Res 37: 145-150, 1995)

GC, gas chromatography

\section{Abbreviations}

${ }^{\mathbf{1}} \mathrm{H}$-MRS, proton magnetic resonance spectroscopy

Cr, creatine

NAA, N-acetyl aspartate

Cho, choline

M-Ino, myo-inositol

PCA, postconceptional age

GA, gestational age

ppm, parts per million

VOI, volume of interest
Measurements of the content of amino acids and related compounds in different anatomical regions of the human brain can elucidate regional brain development and brain pathology $(1,2)$. In most cases, living human brain cannot be sampled for biochemical examination and investigators must rely on in vitro analysis (i.e. HPLC/GC) after brain autopsy. A major problem is to determine whether or not biochemical findings in

Received November 11, 1993; accepted August 25, 1994.

Correspondence: Petra S. Hüppi, M.D., Joint Program in Neonatology, Harvard Medical School, Hunnewell 4, 300 Longwood Ave., Boston, MA 02115.

Supported by Swiss National Foundation (31-30909.91), Maurice Müller Foundation, Milupa SA, and Rossi Fonds. autopsied brain are reasonable representatives of those that prevailed during life. Using ${ }^{1} \mathrm{H}-\mathrm{MRS}$, we have obtained a tool to investigate the chemical composition of the brain in vivo (3-5). However, ${ }^{1} \mathrm{H}-\mathrm{MRS}$ and HPLC/GC have, respectively, inherent limitations and methodologic problems.

The focus of this work is to establish data on regional metabolic changes in early human brain development using a combination of the two methods, one for in vivo assessment $\left({ }^{1} \mathrm{H}-\mathrm{MRS}\right)$ and one for ex vivo measures (HPLC/GC) in autopsy tissue, which should allow us to link the strength of the two tools. The strength of ${ }^{1} \mathrm{H}-\mathrm{MRS}$ compared with HPLC/GC is obviously the data sampling in vivo. 
Although chromatography can be calibrated by standard solutions, determination of absolute concentrations by ${ }^{1} \mathrm{H}$ MRS is still controversial $(6,7)$. Inasmuch as the magnetic resonance spectrum has an arbitrary amplification, several approaches for internal and external standards have been proposed. In an earlier study, we used Cr determined by HPLC in autopsy tissue as an internal standard of our ${ }^{1} \mathrm{H}$-MRS data (8).

HPLC/GC and other in vitro methods are able to detect very low concentrations of numerous metabolites. The concentration of in vivo ${ }^{1} \mathrm{H}$-MRS visible metabolites has to be in the order of $\mathrm{mM}$, thus restricting the number of observable metabolites considerably.

In autopsy, anatomical structures can be respected, whereas in ${ }^{1} \mathrm{H}$-MRS cubes of a minimal size (typically $3.3 \mathrm{~cm}^{3}$ ) have to be used as the VOI. The rectangular shape of the voxel does not fit into anatomical structures, making the amount of unwanted signal even larger. An analysis of the adjacent tissue by HPLC/GC will therefore help to consider this so-called "partial volume effect."

Due to time constraints, only one or two regions of interest can be examined in single-voxel ${ }^{1} \mathrm{H}$-MRS. Using HPLC/GC for the analysis of brain after autopsy, many different anatomical structures can be analyzed at the same time point. Longitudinal studies in the same patient are possible only with in vivo ${ }^{1} \mathrm{H}$-MRS.

To have an additional and methodologically independent assessment of brain metabolite levels during development, we added classic chromatographic data determined in agecorresponding autopsy tissue of different anatomically defined regions of the brain to our in vivo ${ }^{1} \mathrm{H}-\mathrm{MRS}$ study of metabolic brain development.

\section{METHODS}

In vivo data were obtained from a total of 28 newborns, 14 preterm infants (GA 27-34 wk studied at PCA 31-37 wk; postnatal age $18 \pm 10.9 \mathrm{~d}$ ), and 14 infants at term (GA 37-40 wk studied at PCA 38-45 wk; postnatal age $41 \pm 28.9$ d); all newborns were free of cerebral pathology, none of the babies suffered from severe perinatal disorder such as asphyxia or severe respiratory distress syndrome, and none of the babies were ever mechanically ventilated. Approval of the study protocol was obtained by the local ethical committee, and informed parental consent was obtained before study entry.

The autopsy data were obtained from five brains of preterm infants (GA 29.2 $\pm 3.7 \mathrm{wk}$ ) and five brains of term babies (GA $39.6 \pm 1.1$ ); causes of death were congenital diaphragmatic hernia, lung immaturity, and intrapartum (intrauterine) death subpartu due to cord compression. Time between death and autopsy was a few hours in a cooled room $\left(3-4^{\circ} \mathrm{C}\right)$. At autopsy, brain tissue was immediately frozen. With one exception, only half brains were available for determination of regional metabolic mapping; the other half of each brain was used for formalin fixation to determine structural normality. Regional preparation was done by a neuroanatomist.

A 1.5-tesla magnetic resonance unit (GE-SIGNA, General Electric, Milwaukee, WI) was used for imaging and ${ }^{1} \mathrm{H}$-MRS. Preterm babies were kept in the specially designed, tempera- ture-controlled incubator (Spectrospin, Fällanden, Switzerland) during magnetic resonance examination. Cardiorespiratory monitoring was applied to all patients by an integrated monitoring system (Maglife, ODAM-Bruker, Wissembourg, France). Either a surface coil of $7.6 \mathrm{~cm}$ for preterm babies or an extremity coil of $18 \mathrm{~cm}$ for term babies was used for spectroscopy.

A localization image was obtained to position the rectangular VOI in the precentral area of the cerebrum representing the later motor cortex area (Fig. 1A). For the acquisition of the spectra, a stimulated echo pulse sequence was used with repetition time $=$ $2000 \mathrm{~ms}$, echo time $=7 \mathrm{~ms}$, middle interval $=35.5 \mathrm{~ms}$ and complete outside volume presaturation (9). Homogeneity was optimized by shimming to a line width of $3-4 \mathrm{~Hz}$, corresponding to $0.05 \mathrm{ppm}$. Data processing included exponential multiplication $(1 \mathrm{~Hz})$ and a computerized line-fitting routine using Lorentzian line shape. The line-fitting routine of the NAA peak area includes subtraction of underlying glutamine/glutamate signal. Inasmuch as the NAA signal obscures only the right shoulder of the glutamine/glutamate hump, the left shoulder is visible and available for a baseline correction of this spectral region. Assuming an approximately symmetrical shape of the underlying hump, the right shoulder is estimated by a Gaussian line determined by the visible left part of the signal. This completely automated procedure remarkably improved the reproducibility of the determination of the NAA peak area. This algorithm guarantees an operatorunbiased estimation of the baseline in this spectral region and errors introduced by this subtraction are below uncertainties due to other spectrum handling such as phasing.

The quality of our spectra was assured by excluding spectra that contained the following observations: a large signal at $0.9-1.5$ ppm (2 times NAA resonance) representing fat contamination, negative signal at $0.9-1.5 \mathrm{ppm}$ (pulse angle misadjustment), low signal-to-noise ratio (acquisition problems, coil inhomogeneity), broad lines due to shim problems, doubled resonances (motion induced), a large residual water signal determined to be larger than 20 times the $\mathrm{Cr}$ signal (incomplete water suppression), negative peak tails larger than $10 \%$ Cho peak (incorrect phasing), or oscillating artifacts more than $10 \%$ signal (spurious echoes, incomplete water suppression).

All proton spectra included in the study fulfilled the mentioned quality criteria. Samples of in vivo ${ }^{1} \mathrm{H}$-spectra of a preterm infant and term newborn created from a single volume of $3.3 \mathrm{~cm}^{3}$ in the motor cortex area are shown in Figure 1. Metabolite identification was achieved by studies with model solutions at 1.5 tesla and by reference to published information.

Quantification was done by using chromatographically measured $\mathrm{Cr}$ concentrations in region- and age-corresponding human autopsy tissue as an internal standard for the $\mathrm{Cr}$ peak area in each spectrum. This assumes full visibility of $\mathrm{Cr}$ in ${ }^{1} \mathrm{H}$-MRS. To determine the quota of the cytosolic fraction (visible in MRS) of Cr, homogenized brain tissue was centrifuged and $\mathrm{Cr}$ was measured in the supernatant.

For the autopsy data, all regional samples of $1 \mathrm{~g}$ were homogenized, and for $\mathrm{Cr}$ and NAA, HPLC was performed (10). For M-Ino, GC was used for determination of the concentrations (11).

Statistical analysis was done using classic regression analysis. Significance of the slope $>0$ was determined by $t$ test. The level of statistical significance was set at $p<0.05$. Group 


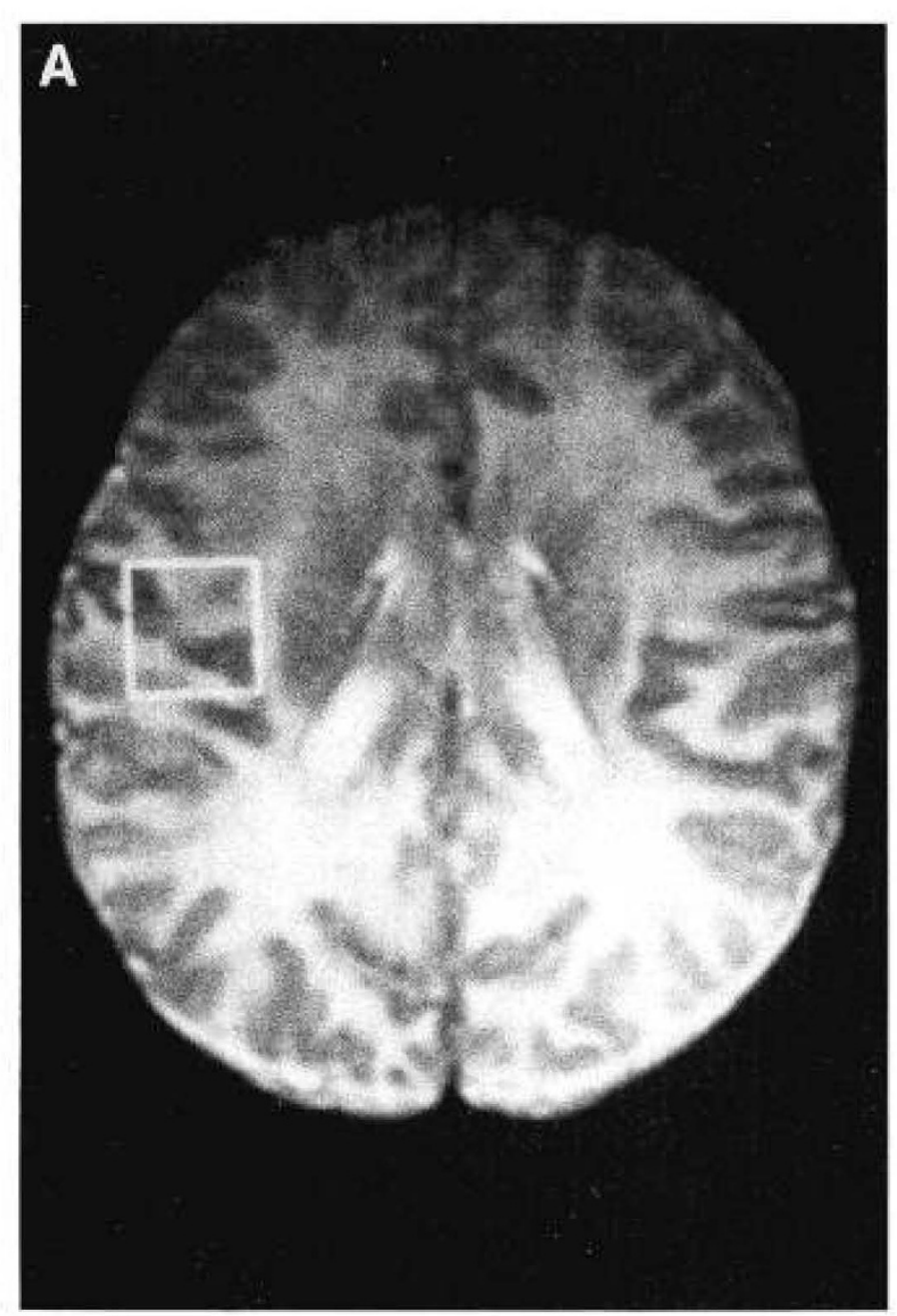

B

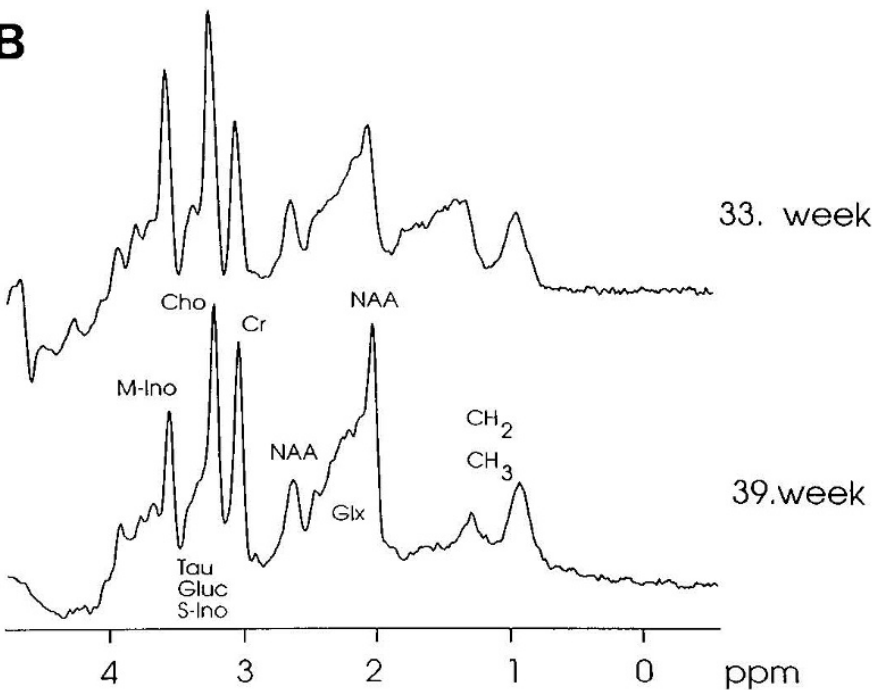

Figure 1. $A$, Localization of the VOI of $3.3 \mathrm{~cm}^{3}$ in the precentral area, shown on a T2-weighted axial magnetic resonance image. $B$, In vivo ${ }^{1} \mathrm{H}-\mathrm{MRS}$ spectra (stimulated echo pulse sequence: echo time $=7 \mathrm{~ms}$, repetition time $=2000$ $\mathrm{ms}$, VOI $=3.3 \mathrm{~cm}^{3}$, precentral area) from a preterm infant at PCA $33 \mathrm{wk}$ and at PCA 39 wk. Resonances assigned: NAA, glutamine/glutamate, Cr, Cho, M-Ino. Taurine ( $\mathrm{Tau}$ ), glucose ( $\mathrm{Gluc}$ ), and scyllo-inositol (S-Ino) contribute to a broad resonance; single resonances cannot be reliably separated. comparison of concentration values in preterm and term newborns was done by $t$ test for independent means.

\section{RESULTS}

Cr determination by HPLC (Table 1). The determination of $\mathrm{Cr}$ in the cytosolic fraction of brain tissue yielded $98 \%$ of total homogenate. Full visibility of $\mathrm{Cr}$ in ${ }^{1} \mathrm{H}$-MRS can therefore be assumed. Evaluation of $\mathrm{Cr}$ concentrations measured by HPLC in different regions of the developing brain showed a significant age-dependent increase that was identical for the left and right side, similar for the precentral area and frontal lobe, and more pronounced for the basal ganglia and thalamus. The regression equation of $\mathrm{Cr}$ in gyrus praecentralis was used for determination of $\mathrm{Cr}$ peak area (internal standard) in the in vivo ${ }^{1} \mathrm{H}$-spectra.

Concentrations determined by in vivo ${ }^{1} H-M R S$ in developing human brain. Age-dependent changes in concentrations of NAA, Cho, and M-Ino in an early stage of human development are given in Figure $2 A-C$. There is a significant increase of NAA by increasing postconceptional age. Looking at effects of postnatal age on in vivo concentrations in preterm infants, M-Ino shows a lower concentration in preterm infants at term compared with term newborns at identical postconceptional age. However, statistical analysis does not prove significance (Table 2).

Comparison of mean concentrations determined by in vivo ${ }^{1} \mathrm{H}$-MRS and HPLC/GC in autopsy tissue of human brain. Calculated mean concentrations values from in vivo ${ }^{1} \mathrm{H}$-MRS for NAA, Cho, and M-Ino in two groups, preterm and infants at term, are given in Table 3 . The significant increase of NAA from preterm to infants at term is confirmed with both methods. M-Ino shows no significant age-dependent (PCA) changes in the examined region. Cho determined only by in vivo ${ }^{1} \mathrm{H}$-MRS remains constant in the two examined age groups.

Concentration values obtained by HPLC/GC in autopsy tissue of the precentral area and by ${ }^{1} \mathrm{H}-\mathrm{MRS}$ in human brain (precentral area) in vivo are comparable in age-dependent changes. NAA concentrations determined in autopsy tissue by HPLC are significantly lower than those measured in vivo by ${ }^{1} \mathrm{H}$-MRS.

Table 1. Statistical evaluation of developmental changes in $\mathrm{Cr}$ concentrations in different regions of brain at autopsy

\begin{tabular}{|c|c|c|c|c|}
\hline & \multicolumn{2}{|c|}{$\begin{array}{l}\text { Single side } \\
\text { regression line }\end{array}$} & \multirow{2}{*}{$\begin{array}{l}\text { Coefficient of } \\
\text { determination } \\
\qquad\left(r^{2}\right)\end{array}$} & \multirow{2}{*}{$\begin{array}{c}p \text { for } \\
\text { slope }>0\end{array}$} \\
\hline & Constant & Slope & & \\
\hline Frontal lobe $(n=12)$ & 2.055 & 0.111 & 0.55 & 0.005 \\
\hline Gyrus praecentralis $(n=11)$ & 1.946 & 0.125 & 0.56 & 0.007 \\
\hline Thalamus $(n=18)$ & -3.678 & 0.335 & 0.60 & 0.0001 \\
\hline Basal ganglia $(n=12)$ & -1.603 & 0.276 & 0.60 & 0.002 \\
\hline
\end{tabular}

Regression lines are defined by constant and slope for each region of the brain. Combined left and right $\mathrm{Cr}$ levels were used to determine the regression line for each region. In 11 half brains, a number of $(n)$ samples were obtained. In all regions, a significant increase ( $p$ for slope $>0$ ) was found (determined from left and right). The regression equation of $\mathrm{Cr}$ in gyrus praecentralis was used for determination of $\mathrm{Cr}$ peak area in the in vivo. ${ }^{1} \mathrm{H}$-spectra (= internal standard). Variation of the data or measure of the goodness of fit is given by the coefficient of determination $\left(r^{2}\right)$. 

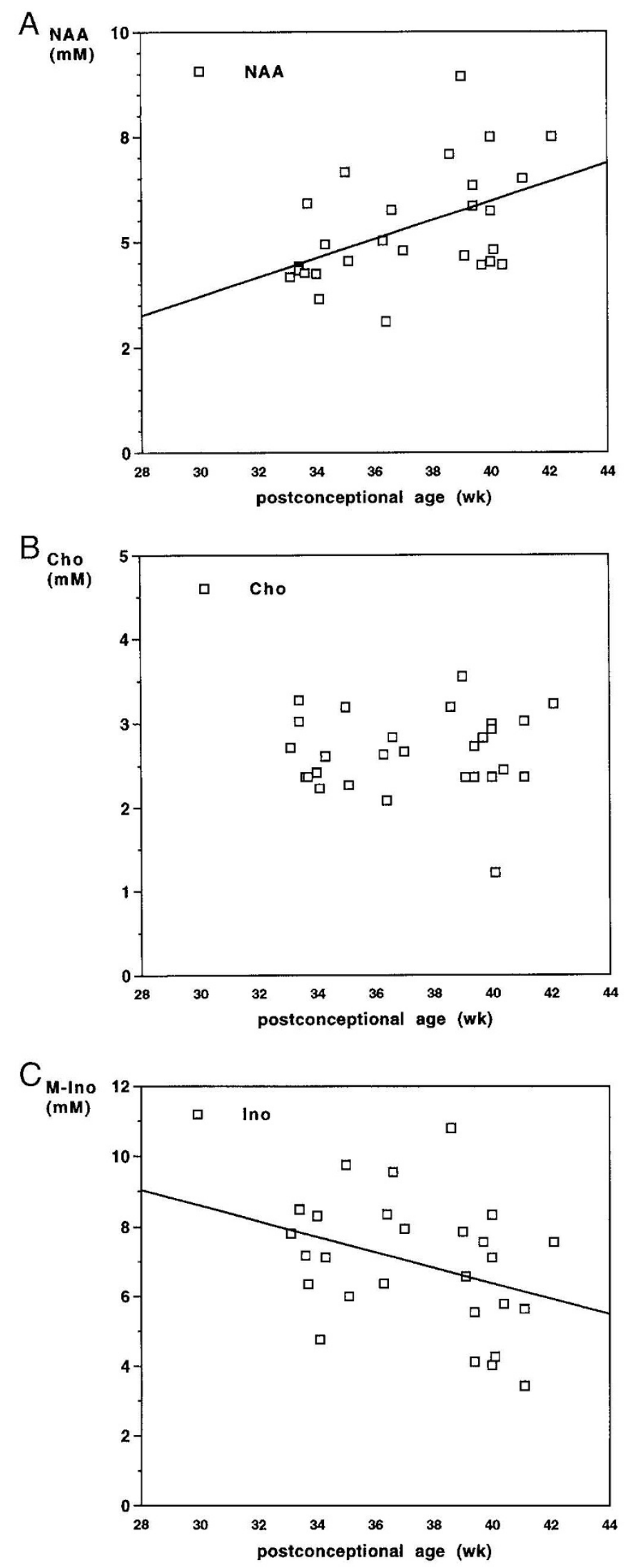

Figure 2. Brain metabolites measured by in vivo ${ }^{1} \mathrm{H}-\mathrm{MRS}$ in early human development. $A$, NAA content is increasing significantly by postconceptional age [regression equation: NAA $=-3.06+0.23 \times \mathrm{PCA}, r=0.5(p=0.01)$ ]; $B$, Cho content is stable during early human development; and $C$, M-Ino content shows no significant change by postconceptional age [regression equation: M-Ino $=15.24-0.22 \times$ PCA, $r=0.35(p=0.07)]$.
Concentrations of NAA, M-Ino, and $\mathrm{Cr}$ in adjacent tissue. To allow consideration of partial volume effects, metabolite concentrations in tissue adjacent to the precentral area were studied by HPLC/GC in anatomically defined regions of autopsy tissue. Concentrations of NAA and M-Ino in the frontal lobe, basal ganglia, and thalamus are given for pooled preterm and term newborns (Table 4). NAA concentration in the precentral area is similar to the concentration in the frontal lobe tissue as in basal ganglia and thalamus in preterm infants. NAA and $\mathrm{Cr}$ concentrations in term newborns are significantly higher in the basal ganglia and thalamus. M-Ino concentrations are higher in the frontal lobe of term newborns than in the precentral area.

\section{DISCUSSION}

Regional ${ }^{1} H$-MRS and contamination from adjacent tissue. To supplement our previously presented data on ${ }^{1} \mathrm{H}-\mathrm{MRS}$ in the cerebellum of preterm and term newborns, we studied regional brain development in the precentral area of the cerebrum, which includes the later motor cortex. Developmental changes are of special importance in the evaluation of the pediatric or neonatal patient. Disease state can only be judged on the basis of normal age-related standards. Localized ${ }^{1} \mathrm{H}-$ MRS has proven to be an acceptable tool for noninvasive study of cerebral development in newborns $(8,12,13)$. To study regional brain development, very small voxels have to be used in the newborn infant; in our study they were as small as 3.3 $\mathrm{cm}^{3}$. However, contamination from adjacent tissue with different metabolite concentrations is still possible. From the results of the regional metabolic assessment in autopsy tissue presented in Table 4, we learn that $\mathrm{Cr}$ concentrations in the thalamus and basal ganglia are much higher than in the precentral area or the frontal lobe; therefore, contamination of the VOI (precentral area) by adjacent tissue from the thalamus and basal ganglia has to be avoided, whereas contamination by frontal lobe would change metabolite concentrations only minimally. This underlines the importance of exact localization and careful selection of control areas in in vivo spectroscopic studies. This comparison of metabolite levels in adjacent anatomical structures of the brain confirms the necessity of exact placement and anatomical shape of the ${ }^{1} \mathrm{H}$-MRS voxel, which is still not satisfactory.

Limitations to absolute quantification in ${ }^{1} H$-MRS. An important feature of our study is the quantitative expression of results. With our method, the chromatographically determined age-corresponding total $\mathrm{Cr}$ concentration of autopsy tissue acts as a reference for the $\mathrm{Cr}$ resonance. $\mathrm{Cr}$ and phosphocreatine are small metabolites, the sum of whose concentration remains constant under different metabolic conditions including $24 \mathrm{~h}$ of brain autolysis. Using this Cr-based method of metabolite quantification, we avoid the necessity of determining the exact water content of the examined brain tissue that is necessary in water signal-based quantification methods (14). Furthermore, cerebrospinal fluid as partial volume does not affect quantification. Petroff et al. (15) have recently shown that the Cr-based quantification method is a valuable technique to study fractionated preparations of brain tissue. We take into account 
Table 2. Concentration of NAA, M-Ino, and Cho measured by in vivo ${ }^{I} H$-MRS; comparison of infants with identical PCA but different postnatal age (PNA)

\begin{tabular}{lccccc}
\hline & PCA (wk) & PNA (d) & NAA (mM) & M-Ino (mM) & Cho (mM) \\
\hline Term newborns (T) $(n=6)$ & $39.9 \pm 1.3$ & $10.5 \pm 2.9$ & $6.7 \pm 1.8$ & $7.6 \pm 1.7$ & $2.9 \pm 0.5$ \\
Preterm at term (PT at T) $(n=8)$ & $39.8 \pm 0.3$ & $62.5 \pm 9.4$ & $5.3 \pm 0.8$ & $5.7 \pm 1.9$ & $2.5 \pm 0.7$ \\
PT at T/T & & $p<0.05$ & $p=0.13$ & $p=0.09$ & $p=0.3$ \\
\hline
\end{tabular}

Comparison was done by $t$ test between preterm infants at term (i.e. $40 \mathrm{wk}$ PCA) and term newborns. There are no significant differences in metabolite concentrations for the studied groups $(n=14)$.

Table 3. Concentrations of NAA, M-Ino, and Cho in precentral area of developing human brain measured in autopsy tissue by $H P L C / G C$ and in vivo by ${ }^{1} H-M R S$

\begin{tabular}{lccc}
\hline & NAA $(\mathrm{mM})$ & M-Ino $(\mathrm{mM})$ & Cho (mM) \\
autopsy/ $/{ }^{1} \mathrm{H}-\mathrm{MRS}$ & autopsy// H-MRS & ${ }^{1} \mathrm{H}-\mathrm{MRS}$ \\
\hline Preterm $(n=5 / 14)$ & $1.2 \pm 0.5 / 4.7 \pm 0.9 p<0.01$ & $8.2 \pm 1.0 / 8.1 \pm 1.5 p=0.85$ & $2.6 \pm 0.4$ \\
Term $(n=5 / 14)$ & $2.8 \pm 0.6 / 6.1 \pm 1.4 p<0.01$ & $7.2 \pm 0.4 / 6.7 \pm 2.2 p=0.63$ & $2.7 \pm 0.6$ \\
Preterm/term & $p<0.01 / p<0.01$ & $p=0.07 / p=0.07$ & $p=0.7$ \\
\hline
\end{tabular}

Comparison was done by $t$ test between preterm and infants at term (age-dependent changes) and between autopsy and in vivo data. Significance is determined at $p<0.05$. GA, PCA, and postnatal age of the two groups are defined in "Methods."

Table 4. Concentrations of NAA, Ino (inositol), and Cr in adjacent tissue (basal ganglia, thalamus, frontal lobe) in autopsy tissue

\begin{tabular}{|c|c|c|c|c|c|c|c|c|c|c|c|c|}
\hline & \multicolumn{3}{|c|}{ Gyrus praecentralis } & \multicolumn{3}{|c|}{ Basal ganglia } & \multicolumn{3}{|c|}{ Thalamus } & \multicolumn{3}{|c|}{ Frontal lobe } \\
\hline & NAA & Ino & $\mathrm{Cr}$ & NAA & Ino & $\mathrm{Cr}$ & NAA & Ino & $\mathrm{Cr}$ & NAA & Ino & $\mathrm{Cr}$ \\
\hline Preterm $(n=5)$ & $1.2 \pm 0.5$ & $8.2 \pm 1.0$ & $5.3 \pm 0.3$ & $1.3 \pm 0.1$ & & $6.1 \pm 1.4$ & $2.2 \pm 0.6$ & & $\underline{6.4 \pm 1.1}$ & $1.2 \pm 0.2$ & $8.9 \pm 0.9$ & $5.4 \pm 0.3$ \\
\hline $\operatorname{Term}(n=5)$ & $2.8 \pm 0.6$ & $7.2 \pm 0.4$ & $7.0 \pm 0.8$ & $3.8 \pm 0.5$ & $6.4 \pm 0.8$ & $9.2 \pm 1.3$ & $4.1 \pm 1.5$ & $7.1 \pm 0.2$ & $9.9 \pm 2.1$ & $2.8 \pm 0.6$ & $\underline{8.7 \pm 0.4}$ & $6.5 \pm 0.6$ \\
\hline
\end{tabular}

Brain tissue from preterm infants (GA $29.2 \pm 3.7 \mathrm{wk}$ ) and from term newborns (GA 39.6 \pm 1.1 ) was used. Underline indicates concentrations with significant $(p<0.05)$ differences to the precentral area. Not enough tissue was available for Ino determination in basal ganglia and thalamus in preterm infants.

age-dependent and regional changes of $\mathrm{Cr}$ that have been shown in the results from the autopsy study. Using this method of quantification, we are able to calculate absolute concentration values for different metabolites but have to consider that they are relative to the age-defined $\mathrm{Cr}$ standard.

Using very short echo times as in this study $(7 \mathrm{~ms})$, differences in T2 tissue relaxation times do not alter signal intensity, and intensity distortion due to J-modulation is reduced. However, contamination of the main spectrum by short $\mathrm{T} 2$ resonances, such as the high molecular weight macromolecules (16) and glutamine/glutamate giving signal around $2.0 \mathrm{ppm}$, could occur. Despite our correction of the baseline in this spectral region, overestimation of NAA concentration in the preterm and term newborn where the overall NAA concentration is low could contribute to differences observed by in vivo measurement by ${ }^{1} \mathrm{H}$-MRS in comparison with in vitro measurement by HPLC (Table 3).

Other confounding factors that have to be avoided are fat contamination, pulse angle misadjustment, low signal-to-noise ratio, shim problems, motion artifacts, incomplete water suppression, incorrect phasing, and spurious echoes. The quality of the spectra used in our study has been tested according the guidelines listed in "Methods" to avoid inclusion of poor data.

Cr determination by HPLC in different regions of brain. $\mathrm{Cr}$ is increasing from preterm to term newborns in all studied regions, but the increase is more pronounced in the thalamus and basal ganglia, where at term $\mathrm{Cr}$ reaches significantly higher levels than in the frontal lobe and the precentral area of the motor cortex (Table 1). Phosphocreatine is a short-term energy storage form of the tissue, and thus the total measured $\mathrm{Cr}$ (sum of phosphocreatine and $\mathrm{Cr}$ ), might well resemble energy potential available in the described area. In their positron emis- sion tomography study, Chugani et al. (17) showed that local cerebral metabolic rates for glucose were highest in thalamus and basal ganglia during very early human brain development. Myelin production in the oligodendroglia is another energydemanding process; subcortical areas are earlier in myelination than cortical structures. The knowledge of the concentration of $\mathrm{Cr}$ in different regions allows use of this metabolite for other studies.

Developmental changes of metabolites measured by ${ }^{1} \mathrm{H}$ $M R S$ and $H P L C / G C$. Increasing interest has been shown toward the substance NAA, a metabolite easily detectable by in vivo ${ }^{1} \mathrm{H}-\mathrm{MRS}$, present largely within neurons (18-20). NAA therefore was used in a wide variety of disease states to document neuronal viability and density, with reduction of NAA suggesting neuronal damage $(21,22)$. Other studies have shown the possible importance of NAA as an acetyl group donor in the formation of brain lipids $(23,24)$.

In this context, it is important to know developmental changes and regional differences of NAA concentration in human brain. Our study shows a significant increase in NAA concentration in the precentral area from healthy preterm infants to term infants. After correction for postconceptional age, postnatal age (d) did not alter NAA concentration significantly when comparing preterm infants at $40 \mathrm{wk}$ PCA with term newborns examined shortly after birth (Table 2). Potential alterations of NAA concentration in neonatal brain due to environmental changes evoked by premature birth and disease state will be studied on the basis of these results.

There is still uncertainty as to which of the Cho-containing compounds contribute to the 3.2-ppm peak seen with ${ }^{1} \mathrm{H}-\mathrm{MRS}$. Free Cho, phosphocholine, and glycerophosphocholine are substances with concentrations below detectability for ${ }^{1} \mathrm{H}$ - 
MRS. It has therefore been postulated that the Cho peak seen with ${ }^{1} \mathrm{H}$-MRS could reflect total brain Cho stores (25). We find stable concentration levels around $3 \mathrm{mM}$ in the developing human brain measured by ${ }^{1} \mathrm{H}$-MRS only, because determination of Cho-containing substances in autopsy tissue can be very diverse. Plasma Cho levels have been shown to be 4 to 7 times higher in newborns than in adults, indicating a high turnover at this time of early human development (26). The resonance observed at $3.58 \mathrm{ppm}$ in the short echo time proton spectrum is attributed to the cyclic sugar alcohol M-Ino.

The M-Ino concentration, assessed by in vivo ${ }^{1} \mathrm{H}-\mathrm{MRS}$, has been shown by Kreis et al. (14) to be decreasing throughout the first year of life. In our study, we can confirm the finding of high M-Ino levels shortly after birth. Comparing M-Ino levels in preterm infants at $40 \mathrm{wk}$ PCA and term newborns, concentration levels seemed to be lower in preterm infants but did not reach significance for the studied number of patients.

Comparison of two methods. Results of NAA and M-Ino concentration determined by ${ }^{1} \mathrm{H}$-MRS were compared with the concentration of NAA and M-Ino measured by HPLC in autopsy tissue. ${ }^{1} \mathrm{H}$-MRS data and chromatographic data from age- and region-corresponding tissue were in agreement for the developmental changes of NAA and M-Ino. Absolute values of NAA showed significantly lower values in autopsy tissue measured by HPLC. This can be in part due to the initial rapid degradation of NAA of approximately $10 \%$ immediately after death (27). After this immediate decrease, postmortem autolysis of NAA occurs very slowly (28). Measurement of NAA concentration by in vivo ${ }^{1} \mathrm{H}$-MRS includes a subtraction of glutamine/glutamate signal, resonances partly overlapping the NAA peak, and subtraction of underlying resonances of macromolecules as mentioned earlier. Therefore, minor contribution of these resonances might cause overestimation of absolute NAA concentration.

The assessment of M-Ino in autopsy tissue and in vivo gave similar concentration values for preterm and term brains. Concentration values have recently been further confirmed by in vivo ${ }^{13} \mathrm{C}$-spectroscopy (29).

Conclusion. Study of regional metabolite concentrations during brain development may help us understand the schedule and organization of the differentiation of brain structures. Although an in vivo observation of low concentration metabolites in small regions of the brain would be desirable, only limited access is possible. Classic biochemical analysis can provide concentration levels of numerous brain metabolites of small samples of brain tissue; however, this is only available in autopsy tissue. The combination of in vivo ${ }^{1} \mathrm{H}-\mathrm{MRS}$ and HPLC/GC in autopsy tissue allowed us to join the strength of both methods and to study regional brain development.

The comparison of two inherently different methods should reduce methodologic artifacts and allows combination of the different findings. In vivo studies of brain development are a basis for better understanding of the physiologic organization of the brain and of pathologic conditions such as abnormal brain development, intrauterine growth retardation, hypoxia, malnutrition, and other factors affecting the newborn.
Acknowledgments. The authors thank Dr. C. T. W. Moonen and Dr. S. Posse for the program source and their help in the implementation and improvement of the stimulated echo pulse sequence, the magnetic resonance team for their technical assistance, Ch. Steffen and A. Jost for their assistance in the biochemical measurements, Dr. G. Gessaga for providing autopsy tissue, and Professor $\mathrm{H}$. Weber for the neuroanatomical preparations.

\section{REFERENCES}

1. Fleming MC, Lowry OH 1966 The measurement of free and $\mathrm{N}$-acetylated aspartic acids in the nervous system. $\mathrm{J}$ Neurochem 13:779-783

2. Okumura N, Otsuki S, Kameyama A 1960 Studies on free amino acids in human brain. J Biochem 47:315-320

3. Radda GK, Rajagopalan B, Taylor DJ 1989 Biochemistry in vivo: an appraisal of clinical magnetic resonance spectroscopy. Magn Reson Q 5:122-151

4. Frahm J, Bruhn H, Gyngell ML, Merboldt KD 1989 Localized proton NMR spectroscopy in different regions of the human brain in vivo. Relaxation times and concentrations of cerebral metabolites. Magn Reson Med 11:47-63

5. Ross B, Kreis R, Ernst T 1992 Clinical tools for the 90s. Magnetic resonance spectroscopy and metabolite imaging. Eur J Radiol 14:128-140

6. Toft PB, Christiansen P, Pryds O, Lou H, Larrson HBW, Stubgaard M, Henriksen O 1992 Absolute concentrations of brain metabolites in childhood measured by ${ }^{1} \mathrm{H}$ magnetic resonance spectroscopy. Society of Magnetic Resonance in Medicine, Proceedings of the 11th Annual Meeting, Vol 1, Berlin, p 2001(abstr)

7. Kreis R, Ernst Th, Ross BD 1993 Absolute quantitation of water and metabolites in the human brain. II. Metabolite concentrations. J Magn Reson 102:9-19

8. Hüppi PS, Posse S, Lazeyras F, Burri R, Bossi E, Herschkowitz N 1991 Magnetic resonance in preterm and term newborns: ${ }^{1} \mathrm{H}$-spectroscopy in developing human brain. Pediatr Res 30:574-578

9. Frahm J, Merboldt KD, Hänicke W 1987 Localized proton spectroscopy using stimulated echoes. J Magn Reson 72:502-508

10. Burri R, Bigler P, Straehl P, Posse S, Colombo JP, Herschkowitz N 1990 Brain development: ${ }^{1} \mathrm{H}$ magnetic resonance spectroscopy of rat brain extracts compared with chromatographic methods. Neurochem Res 15:1009-1016

11. Stokes CE, Hawthorne JN 1987 Reduced phosphoinositide concentrations in anterior temporal cortex of Alzheimer diseased brains. J Neurochem 48:1018-1021

12. Van der Knaap MS, Van der Ground J, Van Rijen PC, Faber JAJ, Valk J, Willemse K 1990 Age-dependent changes in localized proton and phosphorus spectroscopy of the brain. Radiology 176:509-515

13. Peden CJ, Cowan FM, Bryant DJ, Sargentoni J, Cox J, Menon DK, Gadian DG, Bell JD, Dubowitz LM 1990 Proton spectroscopy of the brain in infants. J Comput Assist Tomogr 14:886-894

14. Kreis R, Ernst Th, Ross BD 1993 Development of the human brain: in vivo quantification of metabolite and water content with proton magnetic resonance spectroscopy. Magn Reson Med 30:1-14

15. Petroff OAC, Pleban L, Prichard J 1993 Metabolic assessment of neuron-enrichedfraction of rat cerebrum using high resolution ${ }^{1} \mathrm{H}^{13} \mathrm{C}-\mathrm{NMR}$ spectroscopy. Magn Reson Med 30:559-567

16. Behar KL, Petroff OAC, Spencer DD 1992 Macromolecule resonances in the ${ }^{1} \mathrm{H}-$ NMR spectrum of human brain. Society of Magnetic Resonance in Medicine, Proceedings of the 11th Annual Meeting, Vol 3, Berlin, p 651(abstr)

17. Chugani HT, Phelps ME, Mazziotta JC 1987 Positron emission tomography study of human brain functional development. Ann Neurol 22:487-497

18. Tallan HH 1957 Studies on the distribution of $\mathrm{N}$-acetyl-L-aspartic acid in brain. J Biol Chem 224:41-45

19. Nadler JV, Cooper JR $1972 \mathrm{~N}$-acetyl-aspartic acid content of human neural tumours and bovine peripheral nervous tissue. J Neurochem 19:313-319

20. Koller JK, Zazcek R, Coyle JT $1984 \mathrm{~N}$-acetyl-aspartyl-glutamate: regional levels in rat brain and the effect of brain lesions as determined by a new HPLC method. J Neurochem 43:1136-1142

21. Gill SS, Small RK, Thomas DGT, Patel P 1989 Brain metabolites as ${ }^{1} \mathrm{H}-\mathrm{NMR}$ markers of neuronal and glial disorders. NMR Biomed 2:196-200

22. Menon DK, Baudouin CJ, Tomlinson D, Hoyle C 1990 Proton MR spectroscopy and imaging of the brain in AIDS: evidence of neuronal loss in regions that appear normal with imaging. J Comput Assist Tomogr 14:882-885

23. D'Adamo AF Yatsu FM 1966 Acetate metabolism in the nervous system. N-acetylL-aspartic acid and the biosynthesis of brain lipids. J Neurochem 13:961-965

24. Burri R, Steffen Ch, Herschkowitz N $1991 \mathrm{~N}$-acetyl-aspartate is a major source of acetyl groups for lipid synthesis during rat brain development. Dev Neurosci 13:211-215

25. Miller BL 1991 A review of chemical issues in ${ }^{1} \mathrm{H}$ NMR spectroscopy: N-acetylaspartate, creatine and choline. NMR Biomed 4:47-52

26. Zeisel SH, Epstein MF, Wurtmann 1980 Elevated plasma choline concentration in neonatal plasma. Neurology 30:1226-1229

27. Van Zijl PCM, Moonen CTW 1993 In situ changes in purine nucleotide and N-acetyl concentrations upon inducing global ischemia in cat brain. Magn Reson Med 29:381-385

28. Petroff OA, Ogino T, Alger JR 1988 High resolution proton magnetic resonance spectroscopy of rabbit brain: regional metabolite levels and postmortem changes. J Neurochem 51:163-171

29. Grütter R 1992 Localized ${ }^{13} \mathrm{C}$ NMR spectroscopy of myo-inositol in the human brain in vivo. Magn Reson Med 25:204-210 